\title{
Fuzzy Logic based Greedy Routing (FLGR) in Multi-Hop Vehicular Ad hoc Networks
}

\author{
Shilpy Agrawal ${ }^{1 *}$, R. S. Raw ${ }^{2}$, Neeraj Tyagi ${ }^{1}$ and A. K. Misra ${ }^{1}$ \\ 'Department of Computer Science and Engineering, Motilal Nehru National Institute of Technology, \\ Allahabad - 211004, Uttar Pradesh, India; shilpy15@gmail.com, neeraj@mnnit.ac.in, akm@mnnit.ac.in \\ 2Department of Computer Science and Engineering, Ambedkar Institute of Advanced Communication \\ Technologies and Research, Delhi - 110031, India; rsrao08@yahoo.in
}

\begin{abstract}
In Vehicular Ad Hoc Network (VANET), vehicles on the road provide mainly two types of services to drivers or passengers inside vehicles, namely, safety and comfort services. Safety messages are time-critical, therefore in this paper we have proposed a novel Fuzzy Logic based Greedy Routing (FLGR) protocol which assists in delivering safety messages to the destination vehicle with minimum delay. The proposed FLGR is a multi-hop routing protocol that uses multiple metrics of neighbor vehicles to decide which neighboring vehicle will be the approximate best next-hop node for further forwarding the packets. We have used the concept of fuzzy logic that helps in decision making in VANETs. Fuzzy logic deals with reasoning that is approximate rather than fixed and exact. Fuzzy logic variables may have truth values that range in degrees between 0 and 1 . The fuzzy logic inference system became important and useful when the values of the decision criteria are not only vague but uncertain in nature. FLGR is used within the communication range of the current forwarding node that employs fuzzy logic for selecting the best next-hop node in multi hop VANETs. We have used the fuzzy logic tool box of MATLAB to evaluate our protocol. Metrics such as distance, speed, direction, and position of next-hop forwarding node are put into fuzzy logic system. Thus, our protocol helps in finding the approximate best next-hop node for routing the safety messages by employing fuzzy logic. It selects the next-hop node having maximum distance, speed, and progress and minimum angular deviation of nodes towards destination from current forwarding node. Further, FLGR is more suitable for safety applications in vehicular networks.
\end{abstract}

Keywords: Fuzzification, Fuzzy Logic, Greedy Routing, Membership Functions, Routing Protocol, Vehicular Ad hoc Networks

\section{Introduction}

Wireless network is a kind of infrastructure-less network where communication between the nodes/devices takes place with the aid of radio waves. There are many types of wireless networks, such as wireless mesh networks, Wireless Personal Area Networks (WPANs), Wireless Local Area Networks (WLANs), cellular networks, wireless Mobile Ad hoc Networks (MANETs) etc. Out of these wireless networks MANETs are decentralized kind of wireless networks. It is named so because each node in this network is mobile in nature and is free to move randomly and independently anywhere, in any direction. The network is ad hoc in nature because it does not rely on a pre-existing infrastructure. Each node in the network acts itself as a router to route data packets from one node to the other.

Vehicular Ad hoc Networks (VANETs), is a special class of MANET in which vehicles are the highly mobile nodes. Due to the high speed of vehicles on roads, network topology may change very frequently. However, compare to MANETs, the movement of nodes in VANETs

${ }^{*}$ Author for correspondence 
are restricted to the streets/lanes only where in MANETs nodes can travel anywhere. Vehicular communications in $\mathrm{VANET}^{1}$ has been broadly classified into two types, namely, Vehicle-to-Vehicle (V2V) and Vehicle-to-Infrastructure (V2I) communications as shown in Figure 1. In VANETs each vehicle is termed as a smart vehicle as they are equipped with multi interface cards as well as sensors, both on board and externally. Thus in next few years almost majority of vehicles will be equipped with on-board wireless device, namely On Board Unit (OBU) and Tamper Proof Device (TPD) which has the vehicles secret information. A typical smart vehicle has the following components and technologies, namely, 1. Global Positioning System (GPS) receiver, which enables positioning and navigation services, 2. A wireless transceiver, which helps in V2V and V2I communications, 3. A Central Processing Unit (CPU), which facilitate the implementation of application and communication protocols, and 4 . Various sensors deployed inside and outside of a vehicle which helps in sensing various factors like (speed, acceleration, etc.).

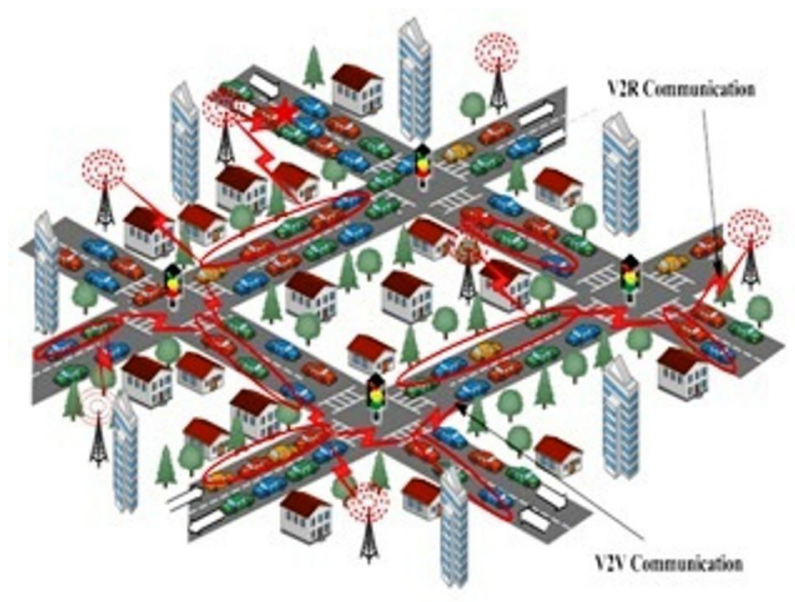

Figure 1. Vanet communications.

Several technologies are explicitly involved in VANETs like GSM, UMTS, and Wi-Max limited Wi-Fi. Wireless Access in Vehicular Environments (WAVE) is the mode of vehicular operations in VANET. It is also known as IEEE $802.11 \mathrm{p}$ wireless standard which is specially meant for VANET. It is a member of IEEE 802.11 family. The recent advancements in the field of ITS has led to the development of Dedicated Short Range Communication (DSRC) protocol, which is designed to endorse high speed, low latency V2V and V2I communications using the WAVE standards ${ }^{2-4}$. Now days VANETs are known for providing three main applications, namely, roadsafety applications, traffic monitoring and management applications, and infotainment services. For the success of any type of above mentioned applications, there is a need of an efficient routing protocol. Routing is the process of finding optimal path and then sending data packet from one node to another until the message eventually reaches the destination node. In VANETs, vehicles may be densely populated on roads in cities or on highways. It may be possible that the source and destination vehicles are at great distance from each other. Therefore, there is a need of intermediate nodes for forwarding messages from source node in the network which are destined for some destination node. The process of routing using intermediate nodes between source and destination is known as multi-hop routing.

There are many types of routing protocols for VANETs like topology based, cluster based, broadcasting based, position based etc. These routing protocols take several aspects into account during route establishment which decide and influence the routing decision. But still there is a need to develop an efficient routing protocol that can deal with highly dynamic nature of the network and imprecise information. Since each vehicle in VANETs is GPS enabled it helps keeping track of its neighbor nodes, therefore position-based routing is the most suitable routing protocol for highly dynamic type of networks such as VANET. As source and destination nodes may be at great distance from each other, therefore selection of next forwarding node by packet carrier node is an essential activity in multi-hop routing.

Since wireless communications are unreliable and vehicles can move at a high speed. Therefore, developing reliable and efficient routing protocol in VANETs is very challenging. Also minimizing end-to-end delay in sending information from source to destination vehicle/s is another major key challenge in VANETs. Therefore, researchers are putting lots of efforts in developing ways which can fulfill these requirements. Academicians have investigated intensely position based greedy routing protocols for VANETs. Research in the area of fuzzy logic decision making system is in boom and many researchers are applying this method in various applications. Also, fuzzy techniques and methods are being employed in the development of intelligent system for decision making, optimization etc. by considering several criterions as an input to fuzzy systems. Wang Xiao-bo et al. ${ }^{5}$ considered two characteristics of VANETs like route life 
time and moving direction of vehicles and employs fuzzy logic to make optimize routing decisions. They also then proposed a novel routing protocol FCAR (Fuzzy Control based AODV Routing) based on traditional AODV. Authors in ${ }^{6}$ have proposed a new protocol that minimizes message overheads by using the concept of fuzzy logic. They have considered three metrics namely, intervehicle distance, node mobility, and signal strength of Current Forwarding Node (CFN) which serves as an input to fuzzy systems. Thus, fuzzy decision making system aids in the selection of next relay node by calculating the fitness value for each neighbor node of CFN and then selects the one which has maximum fitness value. Ghafoor et al. ${ }^{7}$ proposed a new SRR (Stability and Reliability aware Routing) protocol. This protocol exploits fuzzy logic with position based routing to select the best preferable neighbor node around a CFN using distance and direction of neighbor nodes as two input metrics to fuzzy decision making system. Further, the authors have also provided a way that helps the nodes in the network to tackle the network disconnectivity problem. SRR uses a mechanism to cache data packets once the network is disconnected and then switch back to SRR in a connected network scenario.

In this paper, we have explained greedy routing approach which uses a fuzzy logic system to enhance multi-hop routing in VANETs. Fuzzy logic concept helps in achieving dependable solution to route finding. It also helps in selecting the next-hop forwarding node for further transmission in multi-hop VANETs. There are many network simulators like NCTUns ${ }^{8}$, NS-2, NS-3 etc. for evaluating the performance of routing protocols in vehicular networks. In our work, we have used a Fuzzy Logic Toolbox of MATLAB ${ }^{9}$ for evaluating our protocol. The remainder of this paper is organized as follows. Section 2, briefly introduces the basic concepts of fuzzy logic used in this work. Section 3 gives a detail description of the proposed work and section 4 presents the results of simulations done using fuzzy tool box of MATLAB. Finally we present our conclusions in section 5 .

\section{Fuzzy Logic Systems}

Fuzzy logic is a mathematical logic that deals with reasoning that is approximate rather than fixed and exact. Fuzzy logic systems are capable of solving imprecise problems efficiently. It includes fuzzy rules which describe the nodes mobility in an adaptable way with the traffic environment. In fuzzy logic system, we assign multiple values to a wide spectrum of vague data as an input in order to attain the most accurate conclusion possible ${ }^{10}$.There are basically three steps involved as shown in Figure 2 in order to implement fuzzy logic technique to a real life application. These are 1. Fuzzification 2. Fuzzy Inference System (FIS) and 3. Defuzzification.

Fuzzification is the process of converting the classical data also known as crisp data into a linguistic variable using the Membership Functions (MFs) stored in the fuzzy knowledge base. A numerical variable takes some numerical values e.g. speed is $50 \mathrm{~km} / \mathrm{hr}$, whereas a linguistic variable takes linguistic values e.g. speed is very high. These linguistic values constitute a fuzzy set. Thus, linguistic variables are those variables that can take words as its values in natural languages, where these words are characterized by fuzzy sets defined in the Universe of Disclosure in which variable is defined ${ }^{11}$.

Fuzzy Inference System maps inputs by combining a set of membership functions with the control rules to fuzzy outputs. It provides natural thinking mechanism based on human knowledge that include imprecision and ambiguity as shown in Figure 2. The two common types of FIS are Mamdani and Sugeno. In this work, we are using Mamdani fuzzy model. Defuzzification is the process of converting the fuzzy output of the FIS to crisp value using MFs. Different methods like Centroid of Area (COA) or Mean Of Maximum (MOM), Fuzzy Mean (FM) etc. can be used to get a quantifiable result. Our work is focusing on COA method for Defuzzification.

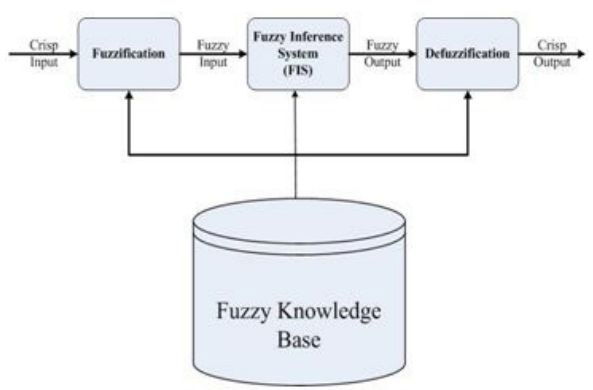

Figure 2. Selection of Node A as next-hop node in FLGR

The major motivation of the fuzzy logic system is to accomplish considerable improvement in the performance of the original greedy routing protocol through the improvement in the procedure of selecting the nexthop forwarding node for further transmission. 


\section{Proposed Work}

There are many types of routing protocols in VANETs as stated above in this paper. But the most suitable one for highly dynamic vehicular networks is a position based greedy routing protocol. Greedy routing protocol is a mathematical process used to ascertain a feasible solution that may or may not be optimal ${ }^{12}$. This strategy is powerful enough for getting a solution that works well for a wide range of applications. Generally, routing protocols based on greedy approach proficiently computes an optimal solution. Therefore, in light of this understanding of greedy approach many routing protocols in highly dynamic networks forwards the packets greedily from one node to another in the network.

In this work, we have assumed that the sending and the receiving vehicles are far away from each other and all the vehicles are GPS enabled. Furthermore, we have used the term vehicle and node interchangeably hereafter. Also, we assume that every node knows its own position through GPS receiver and position of its neighbors by exchanging Hello messages periodically. Therefore, network needed intermediate vehicles which will assist in packet forwarding between source and destination. Furthermore, it is known as position based routing because OBUs are attached on all the vehicular nodes therefore packet carrier node is aware of the position of its neighboring nodes and the position of destination node. In this section, we have proposed the FLGR protocol which incorporates the concept of fuzzy logic for selecting the best next-hop forwarding node.

\subsection{Fuzzy Logic Assisted Greedy Routing (FLGR) Concepts in VANET}

Due to the unique characteristics of the VANETs and being a highly dynamic network, selecting next-hop neighbor node is a challenging task. Therefore, we considered many criteria when we select best next-hop neighbor node in VANETs. We use a Fuzzy Logic assisted Greedy Routing (FLGR) protocol to select the next forwarding node ${ }^{13}$. The selected next-hop forwarding nodes can provide reliable packet forwarding with a high efficiency. In FLGR, the selection of next-hop node for routing depends on multiple metrics of vehicles, namely, its speed, distance from packet carrier node, its direction relative to Current Forwarding Node (CFN) or packet carrier node, its progress towards destination from CFN etc. The fuzzy logic system is a system that provides reasonable decisions based on input membership functions and a group of fuzzy rules similar to the way the human brain operates and thought processes. Therefore, FLGR offers a more logical and consistent way to formalize subjective inputs and perform well in decision making systems, control and prediction processes. FLGR is used to select the next-hop neighbor node intelligently based on above mentioned metrics and thus it facilitate in minimizing delay and sending timecritical safety messages to the neighboring vehicles in the network.

In FLGR, the Current Forwarding Node (CFN) transmits a packet to a destination via intermediate nodes. Therefore, the packet carrier node selects the best nexthop node among various other neighboring nodes from the right half of the circular region as we can see in the Figure 3 by employing the concept of fuzzy logic. During next-hop node selection process we have considered multiple routing metrics such as distance of neighbor node from source, direction, speed, and position towards destination from source for each of the candidate neighbor nodes. These routing metrics are considered as an input of the fuzzy decision making system through which fuzzy output is calculated for each neighbors. The neighbor node with the maximum value of fuzzy output is selected as the best preferable next-hop neighbor node around a source/CFN.

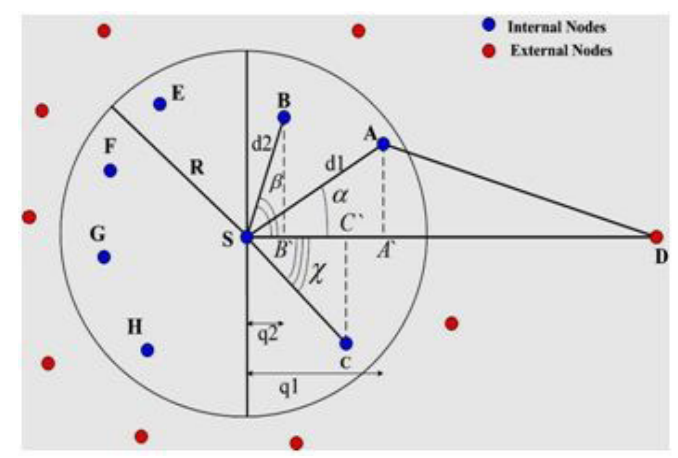

Figure 3. Selection of node a as next-hop node in flgr.

\subsection{Next-hop Node Selection using Fuzzy Logic System}

In FLGR, all the nodes in the network periodically broadcast the Hello packets. A sender node on receiving the Hello packet from the nodes in its transmission range $R$ gets aware of its neighbors. In this case, the source node is unaware that which neighbor node is best next-hop 
neighbor node to forward the packet for the routing purposes. Therefore, fuzzy logic is the efficient solution to this uncertain type of problem. As stated above, four metrics are being considered namely, distance, direction, speed and position of neighbor nodes to opt for the best possible node for further routing the packet. The FLGR is used to select the next-hop forwarding nodes on the routing path through $\mathrm{V} 2 \mathrm{~V}$ communication processes. In this way, a node evaluates the neighbor according to the above metrics. Moreover, through exchanging Hello messages, each node maintains the routing process for each neighbor within the communication range of the forwarding node. For further transmission, these routing results are used.

As shown in Figure 3, FLGR selects the node from the neighboring nodes as the next-hop node which is at maximum distance from the source node and closer to destination node. The next-hop node travels at a very fast speed towards destination, forming minimum angle (between itself, current forwarding node, and destination node), and has maximum progress towards destination from current forwarding node. In the Figure
3 , the current forwarding node is the source node $S$. Source node $S$ has seven neighbors within its transmission range $R$, namely, $A, B, C, E, F, G$, and $H$. These neighbors are internal nodes placed inside the circle. External nodes outside the transmission range (circle) can be used as intermediate node for further transmission. We have discarded nodes $E, F, G$, and $H$ as they are not in the direction of destination node and are far away from the destination too. Thus, we evaluate only the neighbors in the right half of the circular region as shown in the figure above. FLGR selects node $A$ as the next-hop forwarding node as it is positioned at maximum distance from source compare to nodes $B$ and $C$ $(d 1>d 2$ and $d 1>S C)$. It forms smallest angle $A S D(\alpha)$ as compared to angles $B S D(\beta)$ and $C S D(\chi)$ respectively. Also, node $A$ is at maximum speed (assumption) compare to node $B$ and $C$. Furthermore, node $A$ has made maximum progress towards destination from node $S$ as compare to other nodes. The entire proposed work is summarized with the help of an algorithm and flowchart given in section 3.3 and Figure 4 respectively. Section 3.4discusses these metrics in details.

\section{S: Source Node \\ CFN: Current Forwarding Node \\ TN: Total Number of Neighbors of CFN \\ SNN: Selected Next-hop Node \\ BNN: Best Next-hop Node \\ MAX: is a counter \\ Node_id: Vehicle's Identification number}

1. Set $\mathrm{CFN}=\mathrm{S}$

2. Define fuzzy sets for each input and output variables and represent them with membership functions for each input and output metric.

3. Generate fuzzy rules in the form of IF-THEN statements with the help of various routing metrics defined.

4. Set $M A X=0$

5. If destination is in the maximum transmission range of CFN, then exit.

6. For (i=1,node_id $=1 ; \mathrm{i}<=\mathrm{TN} ; \mathrm{i}++$,node_id ++ )

\{

7. Find classical data for the neighbor node i.e. distance from CFN, its direction relative to CFN, its speed and its progress towards destination.

8. Input this real world numerical (classical) data into fuzzy system.

9. Generate fuzzy values by using fuzzy MFs defined in step 2 for each input metrics.

10. Input the fuzzy values obtained in step 9 to fuzzy inference system.

11. Map the fuzzy values to pre-defined IF-THEN rules obtained in step 3 and combine all the rules together to obtain fuzzy output whose value reveals the best next-hop node. 
12. Convert the linguistic result into a numerical value (defuzzify) using the predefined output MF and defuzzification method
13. if (crisp value $>=M A X)$
\{
MAX = crisp value;
SNN = node_id;
\}
\}// end of for loop
14. Set $\mathrm{BNN}=\mathrm{SNN}$
15. Update $\mathrm{CFN}=\mathrm{BNN}$
16. Repeat steps 4 to 15 .
17. End

\subsection{Algorithm for FLGR Protocol}
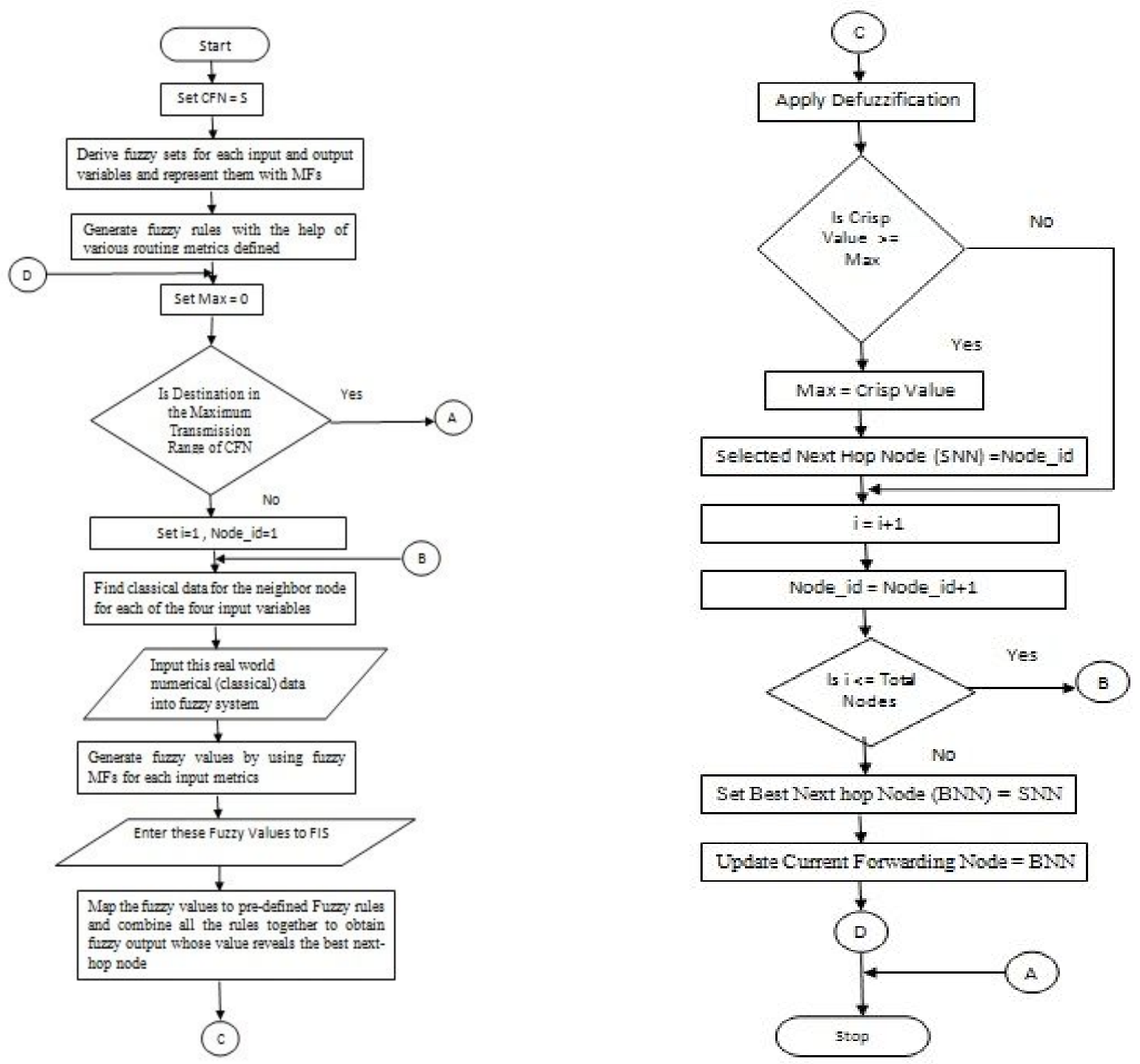

Figure 4. Forwarding technique in FLGR. 


\subsection{Performance Metrics Analysis}

- Distance: A source/CFN upon receiving the Hello packets from its neighbor $A$, calculates the distance between itself and its neighbor $A$. We assume that all the vehicles are having same transmission range $R$ over which stable communications can take place. Therefore, the average distance between the two nodes will be ${ }^{6}$

$$
\operatorname{dist}(A)=\left\{\begin{array}{ll}
\frac{\mathrm{d}}{\mathrm{R}}, & d \leq \mathrm{R} \\
1, & \mathrm{~d}>\mathrm{R}
\end{array}\right\}
$$

Where, $d$ is distance between two nodes as shown in Figure 5. The distance metric indicates the distance of neighbor node from the source node within the transmission range $R$. The higher the value of distance, the more the node is closer to the destination node, thus following greedy method. It means that higher distance leads less number of hops between source and destination nodes. Therefore, FLGR protocol forward traffic safety messages to next neighbors timely.

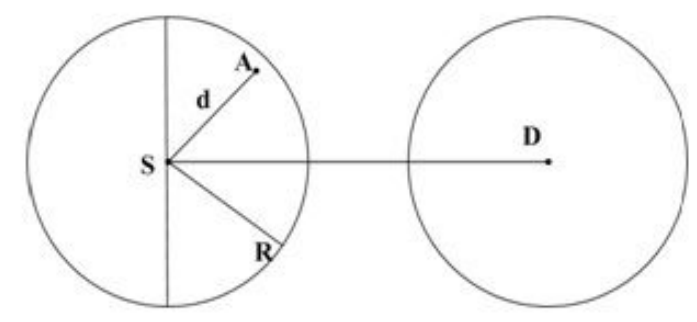

Figure 5. Distance metric calculation.

- Speed: The speed of the vehicles is also one of the dominating factor which helps in deciding in the selection of next-hop forwarding node. In this work, we have assumed the speed of vehicles in the range of $0 \mathrm{~km} / \mathrm{hr}$ to maximum of $100 \mathrm{~km} / \mathrm{hr}$.

- Direction of Next-hop Forwarding Node: This metric also plays a significant role in selecting the best next-hop node. The direction of vehicles is constrained to the roads in vehicular networks. We have assumed that source/CFN and destination are moving in the same direction. $\operatorname{In}^{14-16}$ node $A$ is being selected as a next-hop node as direction $S A$ is more close to direction $S D$ (i.e. angle $A S D$ ) than direction $S B$ as shown in Figure 6. Therefore, angle between the next-hop node, current forwarding node and the destination node (bearing angle) can be calculated as follows ${ }^{17}$

$$
\cos \alpha=\frac{\mathrm{dx}_{\mathrm{s}}\left(\mathrm{dx}_{\mathrm{n}}\right)+\mathrm{dy}_{\mathrm{s}}\left(\mathrm{dy}_{\mathrm{n}}\right)}{\sqrt{\left(\mathrm{dx}_{\mathrm{s}}\right)^{2}+\left(\mathrm{dy}_{\mathrm{s}}\right)^{2}} \cdot \sqrt{\left(\mathrm{dx}_{\mathrm{n}}\right)^{2}+\left(\mathrm{dy}_{\mathrm{n}}\right)^{2}}}
$$

Where, co-ordinates of source node $S$ and node $A$ are $\left(x_{\mathrm{s}}, y_{\mathrm{s}}\right)$ and $\left(x_{\mathrm{n}}, y_{\mathrm{n}}\right)$ respectively.

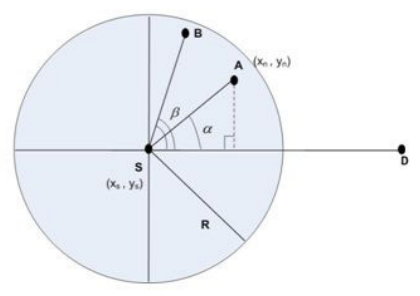

Figure 6. Direction metric representation.

- Position Metric: In VANETs, all the vehicles are GPS enabled and known as smart vehicles. Therefore, it becomes easier to know the position information of neighbor nodes. In this network, each vehicle wishes to know its own position using GPS receiver and position of its neighbors by exchanging Hello message periodically. It means local information like location, direction, speed, and current time can be determined easily. Moreover, source node knows the position of destination node using some location service ${ }^{18}$. Therefore, it becomes easy to compute the position metric for any neighbor node in VANET. In ${ }^{19}$, Position metric $\left(\operatorname{Pos}_{\mathrm{NN}}\right)$ of a neighbor node has been defined as ratio of distance between forwarding node and a point on straight line $S D$ as shown in the Figure7Projection $A$ ' of a neighbor node $A$ lies on the line $S D$ having distances Q.

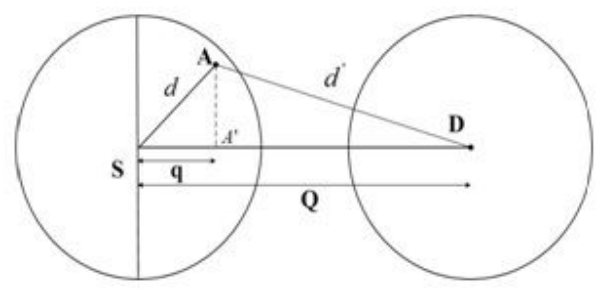

Figure 7. Position metric calculation.

As shown in the Figure 7, the position metric is defined as given below 


$$
\operatorname{Pos}_{N N}=\frac{q}{Q}
$$

Similarly, the progress made by a next-hop node is defined by equation given below

$$
q=\frac{d^{2}+Q^{2}-d^{\prime 2}}{2 Q}
$$

Thus, position metric facilitate in finding the progress of neighbor node towards destination from source node and helps in deciding the best next-hop node for further transmission.

\section{Results Analysis}

Numbers of topology based and position based routing protocols have been proposed for VANETs. Each has its own advantages and disadvantages. Greedy approach based position based routing protocols are found more suitable for VANETs and needs further improvements in these routing protocols. To know their behaviour by considering different metrics in different circumstances we have analysed greedy routing protocol based on Fuzzy inference system.

FLGR protocol uses the fuzzy logic concept in deciding which neighbor node can be selected as the next-hop node to forward the packet from source node to destination node in the networks. In order to develop the system to make routing easier, we have taken four important routing metrics, namely distance, direction, speed and position as inputs variables for Fuzzification. Thus, we have calculated an optimum fuzzy value using MATLAB which decides the best next-hop forwarding node. We have used Gaussian Membership Functions (MFs) in our analysis because it is suitable for highly dynamic random networks such as VANET. These MFs are presented well in the next sub sections.

\subsection{Input Variables for Fuzzification}

All the simulation metrics are illustrated as follows:

- Distance: Here the distance input is classified into different linguistic variables which are defined as follows in Table 1

$$
\operatorname{Gaussian}(x, c, \sigma)=e^{-\frac{1}{2}\left(\frac{x-c}{\sigma}\right)^{2}}
$$

Table 1. Linguistic variables for distance metric

\begin{tabular}{|c|c|}
\hline Very Close & $(0-30$ meters $)$ \\
\hline Close & $(30-75$ meters $)$ \\
\hline Intermediate & $(75-125$ meters $)$ \\
\hline Far & $(125-175$ meters $)$ \\
\hline Very Far & $(175-250$ meters $)$ \\
\hline
\end{tabular}

For each performance metrics, Gaussian membership function is used as defined in equation 5 , based on which five MFs for fuzzy sets are defined as shown in the Figure 8.

Where, $x$ is a linguistic variable, $c$ and $\sigma$ are MFs center and MFs width respectively.

$$
\begin{aligned}
& \text { (i) } \mu_{\text {VeryClose }}(x)=\exp ^{-\frac{1}{2}\left(\frac{x-50}{50}\right)^{2}} \\
& \text { (ii) } \mu_{\text {Close }}(x)=\exp ^{-\frac{1}{2}\left(\frac{x-75}{45}\right)^{2}} \\
& \text { (iii) } \mu_{\text {Intermediate }}(x)=\exp ^{-\frac{1}{2}\left(\frac{x-125}{50}\right)^{2}} \\
& \text { (iv) } \mu_{\text {Far }}(x)=\exp ^{-\frac{1}{2}\left(\frac{x-175}{50}\right)^{2}} \\
& \text { (v) } \mu_{\text {VeryFar }}(x)=\exp ^{-\frac{1}{2}\left(\frac{x-250}{75}\right)^{2}}
\end{aligned}
$$

Figure 8. Membership functions for distance metric.

Further, Figure 9 shows the Gaussian curves for respective membership functions. The source node uses these MFs of distance metric to compute which degree the distance factor belongs to i.e. very close, close, intermediate, far, and very far.

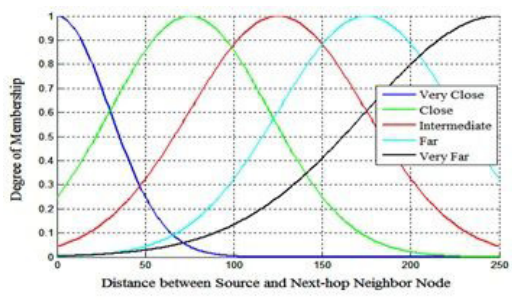

Figure 9. Graphical representation of distance membership function.

- Direction: Similarly, we can define Gaussian membership functions for direction variable. We have taken the range of cos $\alpha$ between 0 and 1 as we have considered only the neighbors in the right half of the circular region i.e. $1^{\text {st }}$ and $4^{\text {th }}$ quadrant only as shown in the Figure 6 . The direction variable is categorized in following three fuzzy sets as shown in Table 2 with their respective membership functions given below in Figure 10. 
Table 2. Linguistic variables for direction metric

\begin{tabular}{|c|c|}
\hline Less directed & $(0-30$ degrees $)$ \\
\hline Mid directed & $(30-65$ degrees $)$ \\
\hline More directed & $(65-90$ degrees $)$ \\
\hline
\end{tabular}

$$
\begin{aligned}
& \text { (i) } \mu_{\text {LessDirected }}(x)=\exp ^{-\frac{1}{2}\left(\frac{x-30}{30}\right)^{2}} \\
& \text { (ii) } \mu_{\text {MidDirected }}(x)=\exp ^{-\frac{1}{2}\left(\frac{x-65}{85}\right)^{2}} \\
& \text { (iii) } \mu_{\text {MoreDirected }}(x)=\exp ^{-\frac{1}{2}\left(\frac{x-90}{25}\right)^{2}}
\end{aligned}
$$

Figure 10. Membership functions for direction of next forwarding node.

The graphical representation of MFs for direction metric is shown in Figure 11. We can easily see the membership degree of direction metric together with the help of membership functions and direction input.

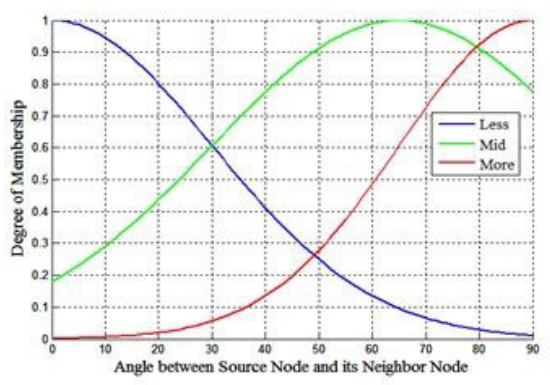

Figure 11. Graphical representation of direction membership function.

- Speed of Neighboring Vehicles: This is an important metric in analyzing the selection of next-hop node while network topology is frequently changing due to highly dynamic nature of vehicular nodes in VANETs. We have classified the speed metric as follows in Table 3.

Table 3. Linguistic variables for speed metric

\begin{tabular}{|c|c|}
\hline Very Low & $(0-20 \mathrm{Kms} / \mathrm{Hr})$ \\
\hline Low & $(20-35 \mathrm{Kms} / \mathrm{Hr})$ \\
\hline Medium & $(35-55 \mathrm{Kms} / \mathrm{Hr})$ \\
\hline High & $(55-75 \mathrm{Kms} / \mathrm{Hr})$ \\
\hline Very high & $(75-100 \mathrm{Kms} / \mathrm{Hr})$ \\
\hline
\end{tabular}

For the above speed classifications the membership functions are defined and graphically shown as under in Figure 12 and in Figure 13 respectively.

(i) $\mu_{\text {VeryLow }}(x)=\exp ^{-\frac{1}{2}\left(\frac{x-20}{20}\right)^{2}}$
(ii) $\mu_{\text {Low }}(x)=\exp ^{-\frac{1}{2}\left(\frac{x-85}{15}\right)^{2}}$
(iii) $\mu_{\text {Medium }}(x)=\exp ^{-\frac{1}{2}\left(\frac{x-55}{20}\right)^{2}}$
(iv) $\mu_{\text {High }}(x)=\exp ^{-\frac{1}{2}\left(\frac{x-75}{20}\right)^{2}}$
(v) $\mu_{\text {VeryHigh }}(x)=\exp ^{-\frac{1}{2}\left(\frac{x-100}{25}\right)^{2}}$

Figure 12. Membership functions for speed metric.

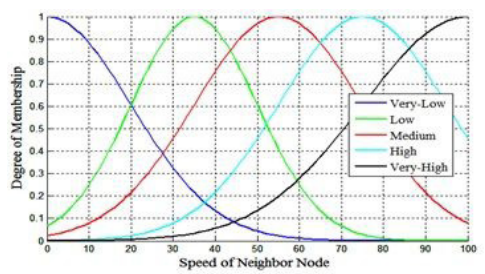

Figure 13. Graphical representation of speed membership function.

- Position Metric: This metric helps in estimating the progress distance of a neighbor node towards destination node by drawing a projection on the line $S D$ as shown in Figure 3. We have classified this metric into four membership functions as shown in Table 4 according to the progress distance from the source vehicle and mathematically presented these MFs in the Figure 14.

Table 4. Linguistic variables for position metric

\begin{tabular}{|c|c|}
\hline Very Close & $(0-30$ meters $)$ \\
\hline Close & $(30-90$ meters $)$ \\
\hline Far & $(90-175$ meters $)$ \\
\hline Very far & $(175-250$ meters $)$ \\
\hline
\end{tabular}

$$
\begin{aligned}
& \text { (i) } \mu_{\text {VeryClose }}(x)=\exp ^{-\frac{1}{2}\left(\frac{x-80}{80}\right)^{2}} \\
& \text { (ii) } \mu_{\text {Close }}(x)=\exp ^{-\frac{1}{2}\left(\frac{x-90}{60}\right)^{2}} \\
& \text { (iii) } \mu_{\text {Far }}(x)=\exp ^{-\frac{1}{2}\left(\frac{x-175}{85}\right)^{2}} \\
& \text { (iv) } \mu_{\text {VeryFar }}(x)=\exp ^{-\frac{1}{2}\left(\frac{x-250}{75}\right)^{2}}
\end{aligned}
$$

Figure 14. Membership functions of position metric.

Figure15 shows the MFs of position metric graphically. Basically, source or CFN uses these membership 
functions of position metric to compute the degree memberships belongs to \{very close, close, far, very far\}.

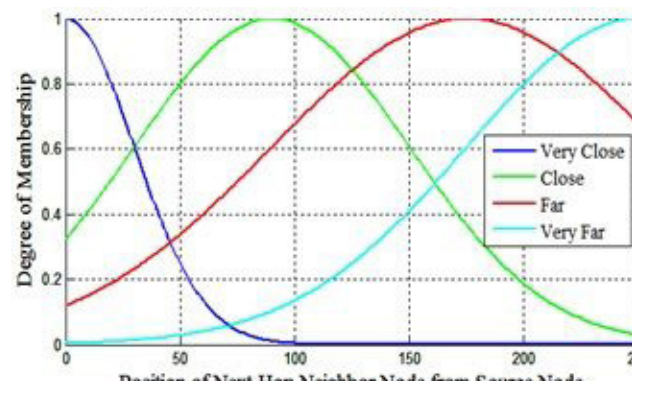

Figure 15. Graphical representation of position membership functions.

\subsection{Output Variable}

The output reflects the features of routing metrics which helps in deciding the best next-hop node for routing. The output optimum function value lies between 0 and 100, and is also defined by Gaussian MF. Thus, the output variable is classified as very low, low, medium, high, and very high as shown in Table 5. The greater the value of this function, the higher the chances of that neighbor node to get selected. The membership functions of above classification are given in Figure16 and its graphical representation in Figure 17 shows the degree of membership of output variable which is calculated using these MFs and output metric.

Table 5. Linguistic variables for output variable

\begin{tabular}{|c|c|}
\hline Very Low & $(0-15)$ \\
\hline Low & $(15-35)$ \\
\hline Medium & $(35-55)$ \\
\hline High & $(55-70)$ \\
\hline Very high & $(70-100)$ \\
\hline
\end{tabular}

$$
\begin{aligned}
& \text { (i) } \mu_{\text {VeryLow }}(x)=\exp ^{-\frac{1}{2}\left(\frac{x-15}{15}\right)^{2}} \\
& \text { (ii) } \mu_{\text {Low }}(x)=\exp ^{-\frac{1}{2}\left(\frac{x-35}{20}\right)^{2}} \\
& \text { (iii) } \mu_{\text {Medium }}(x)=\exp ^{-\frac{1}{2}\left(\frac{x-55}{20}\right)^{2}} \\
& \text { (iv) } \mu_{\text {High }}(x)=\exp ^{-\frac{1}{2}\left(\frac{x-70}{15}\right)^{2}} \\
& \text { (v) } \mu_{\text {VeryHigh }}(x)=\exp ^{-\frac{1}{2}\left(\frac{x-100}{30}\right)^{2}}
\end{aligned}
$$

Figure 16. Membership functions of output variable.

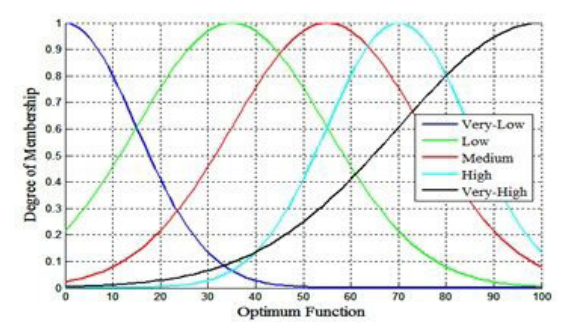

Figure 17. Graphical representation of output membership function.

\subsection{Rule base Fuzzy Inference System (FIS) for Metrics}

In FIS, the human knowledge is represented in terms of fuzzy IF-THEN rules. These rules are designed to bond the input and output variables having in knowledge the routing criteria of greedy based routing protocol. This fuzzy based IF-THEN rule is a conditional statement expressed as ${ }^{11}$

\section{IF $<$ fuzzy preposition $>$, THEN $<$ fuzzy preposition $>$}

Where, IF-part of the rule is known as antecedent or premise and the THEN-part of the rule is known as the consequent or conclusion. Consider Rule 1 of Table 6 . As shown in the table, if distance is Very Far and direction is less and position is Very Far with Very High speed then the fuzzy output is Very High. When the distance of the neighbor vehicle is very large from the source/ CFN within the communication range, then the number of nodes required for forwarding the time-critical safety messages and number of hops can be reduced. Moreover, when the speed of the vehicle is very high, neighbor nodes are more likely to move out of the transmission range but on the other hand are more likely to reach the destination in a short time-span compare to other nodes. This helps in reducing end-to-end delay.

If the neighbor node forms fewer angles with itself, previous node and the destination node then it is more likely to be very far away from the CFN. Thus, its progress towards the destination is very large and helps in reducing the number of hops between source and destination node. In this case, the fuzzy output is defined as very high as given in the table. In this way, keeping into mind all these facts and notions, we have designed rules to get the optimum value of the neighbor nodes. Among these neighboring nodes the node having maximum optimum value will be selected as the next-hop forwarding node. There are 300 possible rules are defined for this work. We 
Table 6. Fuzzy based inference rules

\begin{tabular}{|c|c|c|c|c|c|}
\hline Rules & Distance & Direction (Angle) & Speed & Position & Fuzzy output \\
\hline Rule 1 & Very Far & Less & Very High & Very Far & Very High \\
\hline Rule 2 & Very Far & Less & High & Very Far & Very High \\
\hline Rule 3 & Very Far & Less & Low & Very Far & Low \\
\hline Rule 4 & Very Far & More & Very Low & Very Close & Very Low \\
\hline Rule 5 & Far & Mid & Very High & Far & Very High \\
\hline Rule 6 & Far & Mid & High & Far & Very High \\
\hline Rule 7 & Far & Mid & Medium & Far & High \\
\hline Rule 8 & Far & Mid & Low & Far & Mid \\
\hline Rule 9 & Far & Mid & Very Low & Far & Low \\
\hline Rule 10 & Intermediate & Mid & Very High & Far & High \\
\hline Rule 11 & Intermediate & Mid & High & Far & Mid \\
\hline Rule 12 & Intermediate & Mid & Medium & Far & Low \\
\hline Rule 13 & Intermediate & Mid & Low & Far & Low \\
\hline Rule 14 & Intermediate & Mid & Very Low & Far & Very Low \\
\hline Rule 15 & Close & More & Very High & Close & Mid \\
\hline Rule 16 & Close & More & High & Close & Mid \\
\hline Rule 17 & Close & More & Medium & Close & Low \\
\hline Rule 18 & Close & More & Low & Close & Very Low \\
\hline Rule 19 & Close & More & Very Low & Close & Very Low \\
\hline Rule 20 & Very Close & More & Very High & Very Close & Mid \\
\hline Rule 21 & Very Close & More & High & Very Close & Mid \\
\hline Rule 22 & Very Close & More & Medium & Very Close & Low \\
\hline Rule 23 & Very Close & More & Low & Very Close & Very Low \\
\hline Rule 24 & Very Close & More & Very Low & Very Close & Very Low \\
\hline Rule 25 & Very Far & Less & Medium & Very High & Very High \\
\hline
\end{tabular}

have considered at random any twenty five possible rules as shown in Table 6. Table 7 shows the snapshot of 9 such possible rules using fuzzy tool of MATLAB.

Table 7. Fuzzy Rules

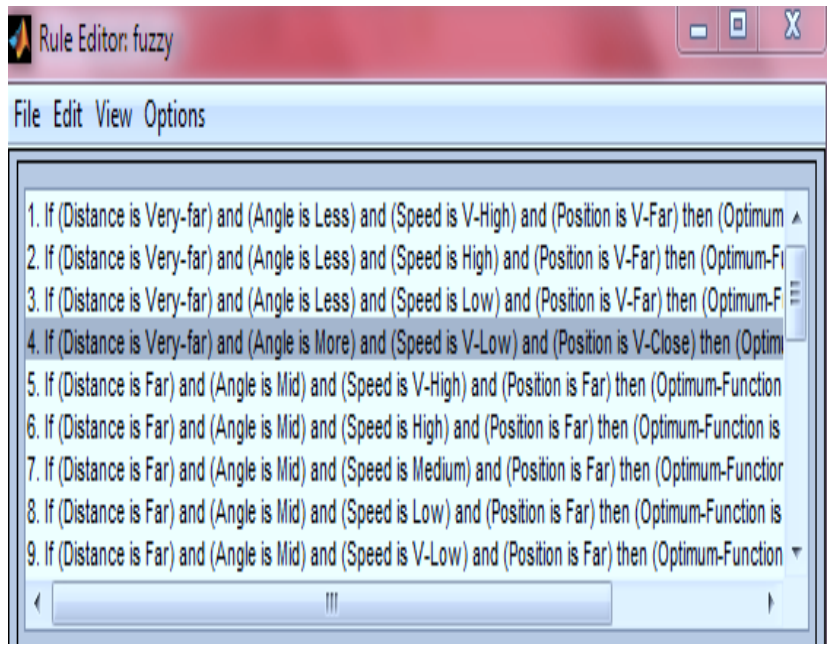

\subsection{Defuzzification}

Defuzzification is a necessary and important process of producing a quantifiable result in fuzzy logic. In the real world, we need a single numerical value as an output to any problem which can be used by any application instead of getting results in linguistic forms like very close, close etc. Therefore, in this work defuzzification has been applied to obtain the crisp value of optimum function. As depicted in above sub section, FIS decisions are based on the testing of all the rules. The rules defined in Table 6 must be somehow combined together to make a decision. Therefore, before defuzzification aggregation process is done in which all the fuzzy sets that represent the outputs of each rule are combined together to obtain a single fuzzy set. Thus, this single fuzzy set obtained serves as an input to a defuzzification process and output of each rule will be the single number. There are many methods of defuzzification. Here we have used the centroid method which is the most widely used method and calculated as 


$$
\operatorname{COA}=\frac{\int \mu(x) x d x}{\int \mu(x) d x}
$$

Where, $x$ denotes fuzzy variable and $\mu(\mathrm{x})$ represents its membership function. It is also known as center of gravity or Center Of Area (COA) method which gives the crisp value of the fuzzy optimum function. Figure18 shows the defuzzified value by a red vertical line in last column and explained in the next sub section through an example.

\subsection{Example Scenario}

In this example, IF distance is very large, speed is very high, angular direction is less and progress distance towards the destination is too large THEN in that case fuzzy optimum function value is high as shown in Figure 18. Thus, the IFpart of the rule "distance is very high" is called the antecedent or premise and the THEN-part of the rule "fuzzy cost is high" is known as the consequent or conclusion ${ }^{9}$. In our simulation work, we have considered a case where distance of candidate node from current forwarding node is 233 meters moving with a speed of $93.2 \mathrm{kms} / \mathrm{hr}$ having angular direction 20.1 degrees and progress towards destination is 190 meters. In this situation the fuzzy output will be 70.5. This output value denotes optimum function value of a specified neighbor node which will be selected as a next-hop forwarding node for further transmission. It reveals that the optimum function increases when distance and speed of neighbor nodes within the transmission range increases, direction get minimized, and progress of nodes towards destination from current forwarding node is more. Thus, in this way we will be able to select that neighbor node as the best next-hop node which has maximum value of this optimum function and therefore helps in reducing the delay in delivering the packets in VANETs.

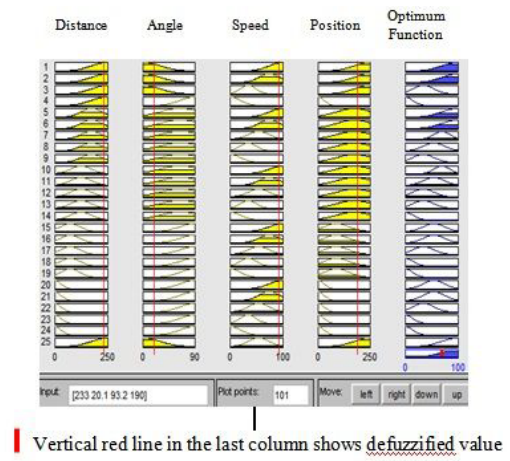

Figure 18. Example of fuzzy inference system.
Furthermore,the FLGR protocol evaluates optimum function values of forwarding nodes considering intervehicle distance, speed, transmission range, received signal strength, angular direction of node, progress distance etc. Three dimensional (3-D) Figures 19, 20, 21 and 22 demonstrate the way fuzzy logic makes inference with the above discussed fuzzy based rules. These figures depict the correlation behaviour between input and output variables. In Figure 19, the 3-D graph shows the value of optimum function that increases when the distance of neighbor node from the source node is more than 190 meters and angle between the source/CFN, neighbor node, and the destination is less than 50 degrees (yellow portion of graph).

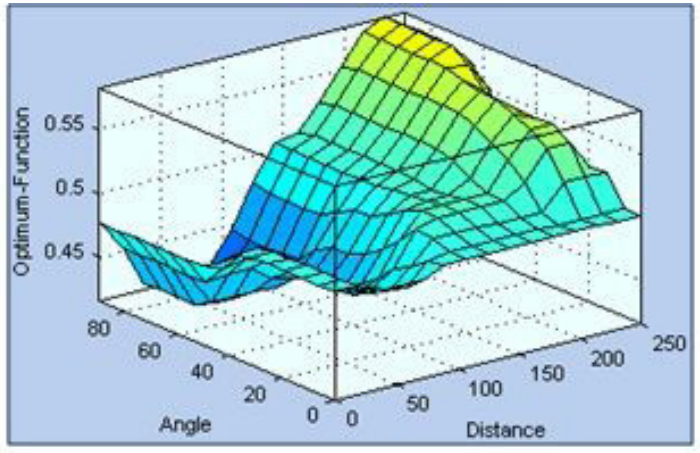

Figure 19. Optimum function for various distances and angles.

Figure 20 shows the correlation behavior between inputs, namely, distance and speed and output optimum function. As the distance and speed of neighbor node from the source/CFN increases, the value of optimum function increases. In this case, the fuzzy output will be very high. This is shown by the dark yellow topmost part of the correlation 3-D graph.

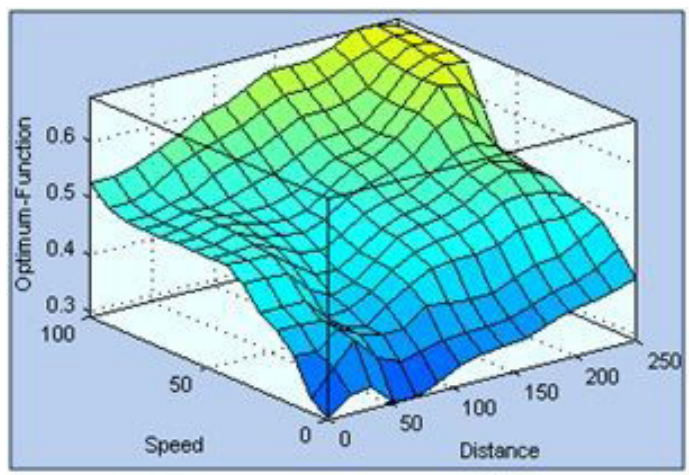

Figure 20. Optimum function for various distances and speed of neighbor node. 
We use Figure21 to show the distribution of optimum function values for speed and relative angles. As shown in the figure, optimum function decreases as the angular direction of neighbouring nodes with respect to line drawn between source and destination increases. For maximum speed towards destination the FLGR has better optimum function and gives better results.

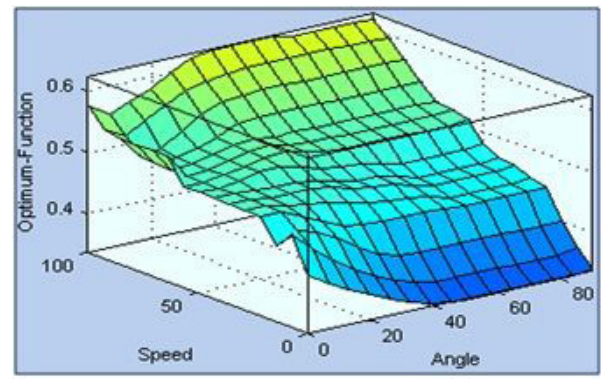

Figure 21. Optimum Function for various angles and speed of neighbor node.

In Figure 22 as both position and speed difference increases the optimum function of fuzzy decision increases (dark yellow topmost part of the correlation 3-D graph). In this case, the path delay between neighbour vehicles increases, but overall end-to-end delay between source and destination node decreases. This is because, as the distance (position) towards destination from current forwarding node increases it leads to lower number of hops between source and destination nodes in the network (dark blue part).

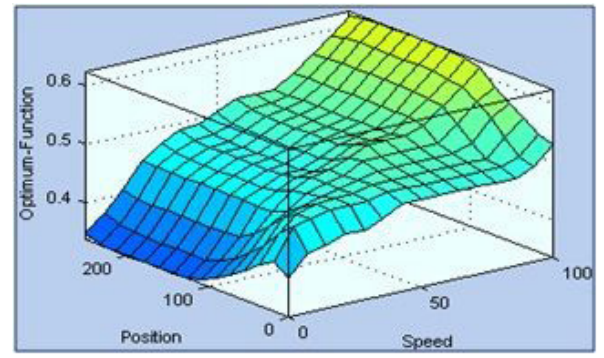

Figure 22. Optimum function for various positions and speed of neighbor node.

\section{Conclusions}

This work focuses on the application of fuzzy logic systems in position based greedy routing approach in VANETs. As fuzzy logic systems are an effective means of conflict resolution of multiple criteria and better assessment of options. In this paper, we have proposed a Fuzzy
Logic based Greedy Routing (FLGR) to select the best next-hop forwarding node for further transmission. The adaptive feature of the FLGR protocol makes it suitable for rapid arrival and departure characteristics of vehicular networks. FLGR considers the percentage of vehicles travelling in the same direction and status of vehicles as inputs of the fuzzy decision making system to tune and optimize the membership functions of the designed fuzzy inference system according to the vehicular traffic characteristics.

The proposed protocol has been designed successfully based on four routing metrics of neighboring vehicles to select the best next-hop node and optimized path. Also, MATLAB simulations were successfully carried out for significant gains of FLGR in terms of fuzzy output as optimum function. Thus it aids in the selection of best next-hop node with minimum delay in various traffic conditions. This work can help those researchers who would like to implement ad hoc networks routing protocols.

\section{References}

1. Karagiannis G, Altintas O, Ekici E, Heijenk G, Jarupan B, Lin K, Weil T. Vehicular networking: A survey and tutorial on requirements, architectures, challenges, standards and solutions, IEEE Communications Surveys and Tutorials. 2011; 13(4):584-616.

2. Ahmed SAM, Ariffin SHS, Fisal N. Overview of Wireless Access in Vehicular Environment (WAVE) Protocols and Standards. Indian Journal of Science and Technology. 2013 Jul; 6(7):4994-5001.

3. Jiang D, Delgrossi L. IEEE 802.11p: Towards an International Standard for Wireless Access in Vehicular Environments. IEEE Vehicular Technology Conference; Singapore. 2008 May 11-14. p. 2036-40.

4. Vegni AM, Biagi M, Cusani R. Smart Vehicles, Technologies, and Main Applications in Vehicular Ad hoc Networks [internet].Vehicular Technologies - Deployment and Applications, Dr. Lorenzo Galati Giordano (Ed.), ISBN: 978-953-51-0992-1, InTech, DOI: 10.5772/55492. Available from: http://www.intechopen.com/books/ vehicular-technologies-deployment-and-applications/ smart-vehicles-technologies-and-main-applications-invehicular-ad-hoc-networks

5. Wang X, Yang Y, An J. Multi-Metric Routing Decisions in VANET. The Eighth IEEE International Conference on Dependable, Autonomic and Secure Computing; Chengdu. IEEE; 2009 Dec 12-14. p. 551-6. 
6. Wu C, Ohzahata S, Kato T. Fuzzy Logic Based Multi-Hop Broadcast for High-Density Vehicular Ad Hoc Networks. Vehicular Networking Conference; NJ. IEEE; 2010 Dec 13-15. p. 17-24.

7. Ghafoor KZ, Bakar KA, Salleh S, Lee KC, Mohamad MM, Kamat M, Arshad MM. Fuzzy Logic-Assisted Geographical Routing Over Vehicular Ad Hoc Networks. International Journal of Innovative Computing, Information and Control. 2012 Jul; 8(7B):5095-120.

8. Gamess E, Chachati M. Analyzing Routing Protocol Performance with NCTUns for Vehicular Networks. Indian Journal of Science and Technology. 2014 Sep; 7(9):1391-402.

9. Available from: www.mathsworks.in/help/fuzzy/foundations-of-fuzzy-logic.html\#bp78170-7; [cited 2014 Nov 11].

10. Klier GJ, Yan B. Fuzzy Sets and Fuzzy Logic Theory and Applications. NJ: Prentice Hall; 1995.

11. Wang Li-Xin. A Course in Fuzzy Systems and Control. Prentice Hall International, Inc; 1997.

12. Sanjiv. The Greedy Method [Internet]. Available from: www.cs.umsl.edu/ sanjiv/classes/cs5130/lectures/gm.pdf; [cited 2014 Oct 15].

13. Shilpy A, Raw RS, Neeraj T. Enhancing Greedy Routing using Fuzzy Logic for Vehicular Ad hoc Networks.
Proceedings of the International Conference on Advances in Computing, Control and Networking-ACCN 2015; Bangkok, Thailand; 2015 Feb 21-22. p. 98-102.

14 Stojmenovic I. Position-Based Routing in Ad Hoc Networks. Communication Magazine, IEEE. 2002 Jul; 40(7):128-34.

15. Kranakis E, Singh H, Urrutia J. Compass routing on geometric networks. In: Proc 11th Canadian Conference on Computational Geometry, Vancouver; 1999.

16. Takagi $\mathrm{H}$, Kleinrock L. Optimal transmission ranges for randomly distributed packet radio terminals. IEEE Transactions on Communications. 1984; 32(3):246-57.

17. Ghafoor KZ, Bakar KA, Lloret J, Khokhar RH, Lee KC. Intelligent beaconless geographical forwarding for urban vehicular environments. Wireless Networks. 2013 Apr; 19(3):345-62.

18. Camp T, Boleng J, Wilcox L. Location Information Services in Mobile Ad hoc Networks. IEEE International Conference on Communications (ICC); IEEE; 2002. p. 3318-24.

19. Raw RS, Lobiyal DK. Improved Position-Based Routing in Vehicular Ad hoc Networks using P-DIR Method. Advances in Computing and Communications (ACC); Berlin Heidelberg. Springer; 2011; 192(Part III):654-63. 